\title{
Intelligence-driven mobile networks for smart cities
}

\author{
Liang Zhao ${ }^{1}$. Jia $\mathrm{Hu}^{2} \cdot$ Ahmed Al-Dubai $^{3}$. Celimuge $\mathrm{Wu}^{4}$ - Qi Wang ${ }^{5}$. \\ Ammar Hawbani ${ }^{6}$ \\ Published online: 8 January 2021 \\ () The Author(s), under exclusive licence to Springer-Verlag GmbH, AT part of Springer Nature 2021
}

For the last twenty years, artificial intelligence (AI) has become a significant technology to influence our daily life with its ability of promoting the quality of living. AI can find the patterns and create actions from the proper amount of data, then make proper decisions for applications. It is promising to involve $\mathrm{AI}$ in the smart cities to enable the better intelligent services. Among all services, mobile networking can be also improved by applying AI, in which such enhanced networking will allow broad coverage, high capacity, high resource availability, and extensive connectivity. It will meet the key metrics including bandwidth, jitter, throughput, transmission delay, and availability.

Therefore, this special issue is vital to foster the application and innovation of AI and ML technologies for mobile networking within 5G, MANETs, VANETs, wireless sensor networks and as well as other forms of networks. This is to explore a way

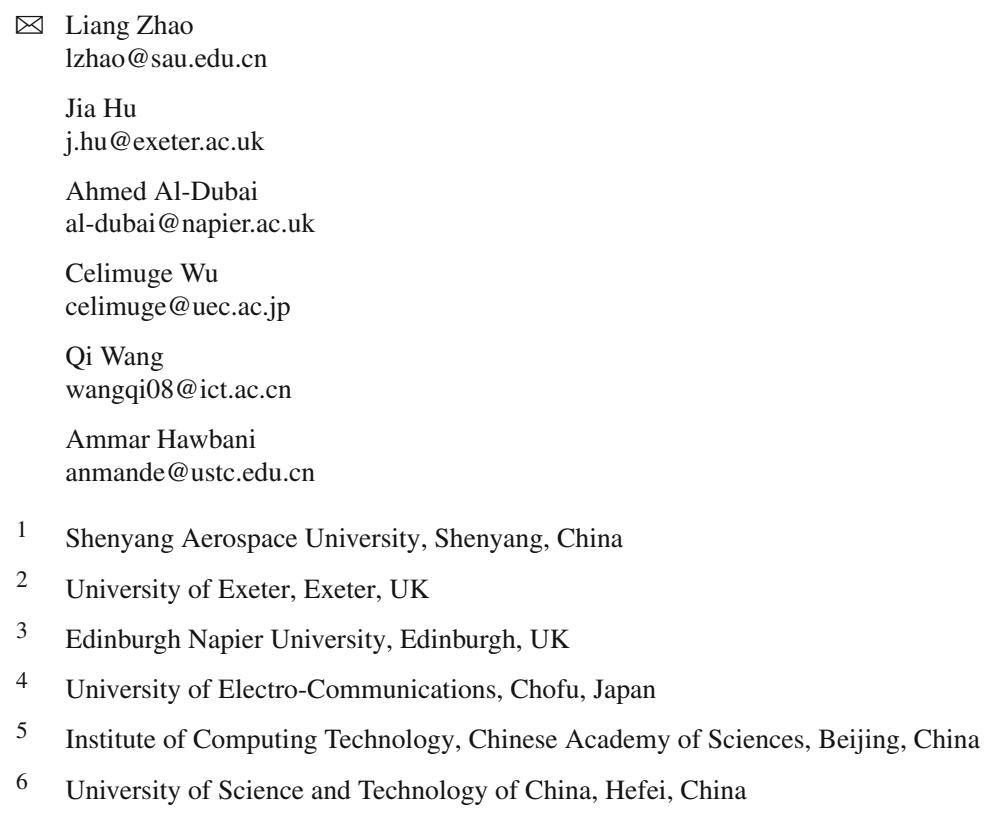


of seamless and intelligence-driven communications for enabling evolution of the current networks and beyond, and thus, to support the sustainable smart cities. In this special issue, we have received 22 submissions, of which 8 papers were selected for publication after the process of strict peer review. We show the details of our selected papers as follows.

Mobile Networking is the first thing to concern for smart cities. The paper entitled "A Self-Adaptive Network for Multi-Robot Warehouse Communication" provided by Varma et al., discusses the communications for multi-robot warehouse within the concept of smart warehouse. A new model, namely, Transmission Deadline and Information Content based Priority, is proposed to improve transmission success rate. In order to achieve this, path planning of the robots is also considered for reducing the outage probability of the failed transmission. The paper entitled "Delegation of Authentication to the Data Plane in Software-Defined Networks" provided by Almaini et al., presents a work to tackle the drawbacks of support-less of new header definitions. As claimed by the authors, this work is the first to proposed the delegation of typical security functions from the intelligent controller to the data plane. The experimental results also show the proposed solutions improve the network performance by offloading the duty of controllers. In addition, the paper entitled "LSTM based link quality confidence interval boundary prediction for wireless communication in smart grid" provided by sun et al., studies the link quality prediction of the wireless communication in smart grid. In particular, a Long-Short-Term-Memory (LSTM) based link quality confidence interval boundary prediction is proposed in order to provide accurate and trustworthy link prediction. Besides, as a fundamental part of mobile networking, the shortest path problem is also studied in this special issue. The paper entitled "Diversity Metrics for Direct-Coded Variable-Length Chromosome Shortest Path Problem Evolutionary Algorithms" provided by Ghannami, studies the genetic algorithm (GA) for solving the shortest path problem. In particular, the authors proposes a chromosome-length-based metric to measure the chromosome-length diversity of GA. Principal Component Analysis (PCA) is also applied to analyse the correlations between the proposed metrics with different size of network/population sizes.

Mobile Social Network is an essential component in smart cities supported by the intelligent mobile networking. The paper entitled "Efficient Service Discovery in Mobile Social Networks for Smart Cities" provided by Guo et al., introduces an efficient service discovery model, namely, friends' cycle service discovery (FCSD), to enhance the interaction among citizens in the smart cities. In FCSD, intelligent network nodes with common social interests can interact and form social cycles self-organized. Moreover, The paper entitled "RNe2Vec: Information Diffusion Popularity Prediction based on Repost Network Embedding" provided by Shang et al., also focuses on the mobile social networks where it intends to eliminate the dependency of human experience and embedding learning during the popularity prediction. Hence, they propose a repost network embedding-based diffusion popularity prediction algorithm for achieving the precise prediction.

Intelligent Transport System can be also accomplished by equipping efficient mobile networks, so as to build the smart cities. The paper entitled "A Method of Chained Recommendation for Charging Piles in Internet of Vehicles" provided by Zhang et al., investigates the recommendation of charging tiles in the Internet of Vehi- 
cles (IoVs). The authors proposes a new recommendation method by considering the user's warning level and the real-time state of electric vehicles and charging piles. The intelligent selection of charging piles reduces the conflicts of recommendation and provide personalized service for the networked vehicular users. In addition, the paper entitled "An Improved YOLO-based Road Traffic Monitoring System" provided by Al-qaness et al., presents an intelligent video surveillance-based system for tracking the vehicles. The proposed method is obtained by training different datasets where real traffic video is also applied to examine the performance of the proposed system.

Publisher's Note Springer Nature remains neutral with regard to jurisdictional claims in published maps and institutional affiliations. 